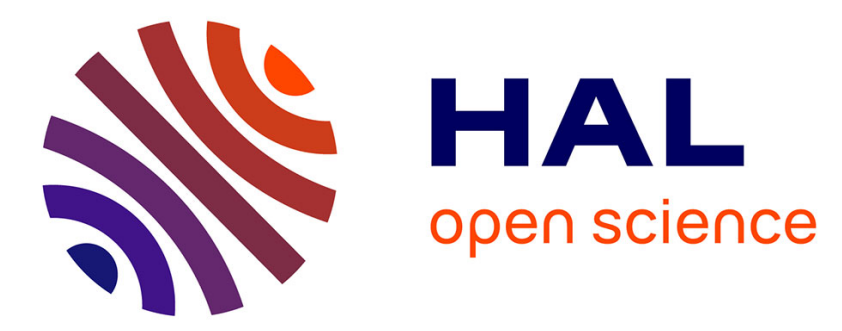

\title{
IRIS IDENTIFICATION AND ROBUSTNESS EVALUATION OF A WAVELET PACKETS BASED ALGORITHM
}

Florence Rossant, Manuel Torres Eslava, Thomas Ea, Frédéric Amiel, Amara Amara

\section{To cite this version:}

Florence Rossant, Manuel Torres Eslava, Thomas Ea, Frédéric Amiel, Amara Amara. IRIS IDENTIFICATION AND ROBUSTNESS EVALUATION OF A WAVELET PACKETS BASED ALGORITHM. ICIP, Sep 2005, Genoa, Italy. 10.1109/ICIP.2005.1530377 . hal-02882948

\section{HAL Id: hal-02882948 https://hal.science/hal-02882948}

Submitted on 28 Jun 2020

HAL is a multi-disciplinary open access archive for the deposit and dissemination of scientific research documents, whether they are published or not. The documents may come from teaching and research institutions in France or abroad, or from public or private research centers.
L'archive ouverte pluridisciplinaire HAL, est destinée au dépôt et à la diffusion de documents scientifiques de niveau recherche, publiés ou non, émanant des établissements d'enseignement et de recherche français ou étrangers, des laboratoires publics ou privés. 


\title{
IRIS IDENTIFICATION AND ROBUSTNESS EVALUATION OF A WAVELET PACKETS BASED ALGORITHM
}

\author{
Florence ROSSANT, Manuel TORRES ESLAVA, Thomas EA, Frédéric AMIEL, Amara AMARA
}

\author{
Institut Supérieur d'Electronique de Paris, ISEP \\ 21, rue d'Assas \\ 75006 PARIS, France \\ florence.rossant|manuel.torres-eslava|thomas.ea|frederic.amiel|amara.amara@isep.fr
}

\begin{abstract}
This paper presents an iris recognition system, based on a wavelet packet analysis using orthogonal wavelets. The identification of the different packets that carry discriminating information about the iris texture is carried out through an energy measure. Tests, conducted on a database of 149 high quality iris images show good robustness in relation to changes in illumination, blurring, optical axis deviation or local defects in the images.
\end{abstract}

\section{INTRODUCTION}

Importance of biometric identification is increasing everyday. Nowadays, traditional methods for personal identification (a physical key, ID card, a secret password, etc.) are being replaced or augmented by biometric methods (iris texture, fingerprint, face, etc.) in areas of high security.

Iris recognition technology has been a field of intensive research for the last 15 years. The first system was patented by Daugman in 1994 [1]. Daugman's system is based on non-orthogonal wavelet analysis using so called Gabor wavelets [2], and has emerged as something of a benchmark in the field. Since then, much further work has been done by several research groups such as Tan et al. [3,4], Tisse et al. [5], Wildes [6], Sanchez et al. [7]. Lim et al. [8] have proposed a system based on orthogonal wavelet transform using the Haar wavelet. It uses the diagonal detail $(\mathrm{HH})$ coefficients as iris signature. However, the wavelet packet approach can be a good alternative to the standard wavelet transform since it offers a more detailed division of the frequency plane [9]. It has been tested successfully in case of images acquired in visible light illumination $[10,11]$.

In this paper, we present an algorithm based on wavelet packet decomposition and put the emphasis on some improvements realized with respect to [10]. We also show that our algorithm is robust with regards to acquisition conditions, by simulating changes in illumination, blurring, optical axis deviation, or by adding local defects. These tests enable us to determine precisely the range of image degradations that can be allowed without decreasing the identification rate and to calibrate the image acquisition system.

\section{IRIS EXTRACTION}

The images used for this study were acquired by Miles Research [12] using dedicated iris imaging equipment providing very little eyelid and eyelash occlusion and also little light reflections. The images were resized to $600 \times 400$ pixels. The localization process finds the iris boundaries (Fig. 1a). Then, the iris texture is transformed into a polar representation and the contrast of the iris texture is increased by histogram equalization (Fig. 1b). (a)

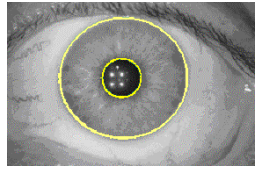

(b)

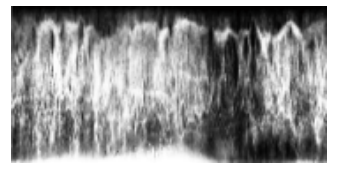

Figure 1. a) Iris localisation, b) unwrapped iris texture after histogram equalization

\subsection{Localization and equalization}

The robustness and the accuracy of the localization process [10] have been improved. The algorithm is based on the model of the iris: the pupil boundary is a circle, the external iris boundary is an ellipse, the center of the ellipse is closed to the center of the circle. It also uses the fact that there is some contrast between the iris and the surrounding white sclera tissue or the pupil. There are four steps (Fig. 2). The first pre-processing step is inherent to the illumination system used for image acquisition: it detects and filters the four bright spots located inside or sometimes near the pupil (Fig. 2a, 2b). In the second step, we use histogram analysis to detect the darkest pixels in a sub-window at the center of the image. 
Thus, we find approximately the pupil (Fig. 2c). From this information, we deduce a grid of possible locations for the pupil center. In the third step, we find the center and the radius of the circle that maximize the mean gradient in the orthogonal direction of the curve (Fig. 2d). A similar process is used in the fourth step for determining the ellipse. Because of possible eyelid or eyelash occlusion, we limit the gradient maximization to left and right subpart of the ellipse (Fig. 2e). This algorithm has proved to give very good results, even in case of very low contrast, because of the average effect, and because there is no need of parameter tuning as we use a maximization criterion. (a)

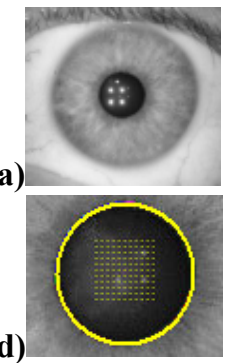

(b)

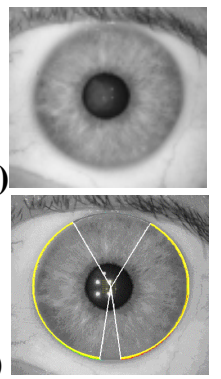

(c)

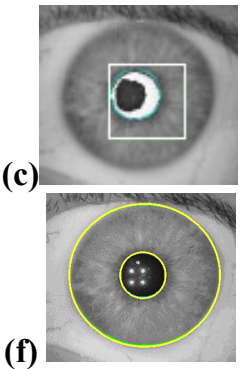

Figure 2. a) Part of an original eye photo, b) spot filtering, c) coarse pupil localization, d) grid of possible centers and pupil detection, e) external boundary detection with angle limitations, f) final result

\subsection{Unwrapping}

We use the adapted polar transform that was suggested by Daugman [2]. The transform is expressed by (1), where $\left(x_{p}, y_{p}\right)$ and $\left(x_{i}, y_{i}\right)$ are a pair of coordinates on the pupil and iris border. The figure to the left of Equation (1) defines the angle variable $\theta$ and the radius variable $r$. The radius $r$ is normalized to the interval $[0,1]$.

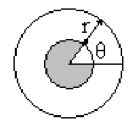

$$
\begin{aligned}
& x(r, \theta)=r x_{i}(\theta)+(1-r) r x_{p}(\theta) \\
& y(r, \theta)=r y_{i}(\theta)+(1-r) r y_{p}(\theta)
\end{aligned}
$$

The size of the unwrapped images is set to $256 * 128$ pixels. The last step is a histogram equalization to increase the contrast of the texture (see Fig. 1b).

\section{IDENTIFICATION ALGORITHM}

The algorithm is based on a signature extraction and a distance measure to compare signatures.

\subsection{Signature extraction}

We use the Biorthogonal 1.3 wavelet in a 3-level wavelet packet decomposition to extract the texture features of the unwrapped images [10]. This generates 64 output subimages (wavelet packets), numbered 0 to 63 , each holding information about both frequency and localization. We use an energy measure to evaluate which packets carry the most texture information:

$$
E_{i}=\sum_{j, k} w_{i}(j, k)^{2}, i=0, \ldots, 63
$$

where $E_{i}$ is the energy of the sub-image $w_{i}$. Fig. 3 plots the mean energy distribution for the 149 unwrapped images of our iris base. This figure shows that wavelet packets 2 and 10 , and also 8 are the most suitable to use as signature. Packet 0 is not considered since it only contains DC information.

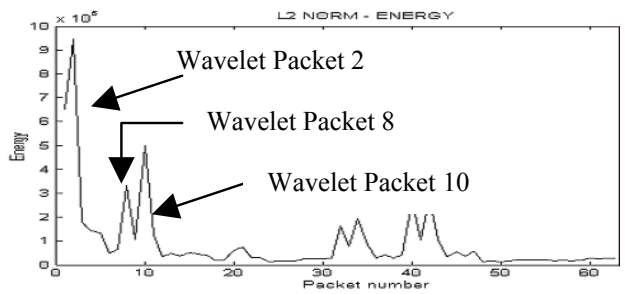

Figure 3. Mean wavelet packet energy distribution

A compact signature was first achieved [10] by quantizing the wavelet coefficients into one bit each, depending on their sign. Packets 2,8 and 10 are now encoded using (3):

$s_{i}(j, k)=\operatorname{sign}\left(w_{i}(j, k)\right) \quad$ if $\left|w_{i}(j, k)\right|>S$

$s_{i}(j, k)=0$ otherwise

with threshold $S=10$ optimised experimentally. We refer here to image de-noising using wavelets: small detailed coefficients are put to zero, before applying the inverse transformation [13]. In our application, we consider that small coefficients are not significant, and we use this encoding to decrease their impact in the distance measure. Thus, we obtain for each packet a compact signature of $\mathrm{N}=16 * 32$ coefficients equal to $-1,0$ or 1 .

\subsection{Distance measure and discrimination results}

We compute the distance between two signatures in order to decide if both come from images of the same iris (authentic distance) or from images of different iris (imposter distance). The new distance measure is expressed in Equation (4):

$H D_{i}=\frac{1}{2 N} \sum_{j, k}\left|s_{i}{ }^{(1)}(j, k)-s_{i}{ }^{(2)}(j, k)\right|$

where $s_{i}^{(1)}$ and $s_{i}^{(2)}$ are two signatures obtained from packet $i$. In order to verify that discrimination is properly achieved, we compute this distance between each signature and all the others. We define the separation as the distance between the largest authentic measurement and the smallest imposter measurement. The separation should be positive.

The tests were conducted on 149 images of 69 
different eyes. Thus there was more than one image per each eye. Fig. 4 displays the results obtained for packet 2, and shows that the separation is positive $(0.082)$ and has been increased by $8 \%$ compared to the results in [10].

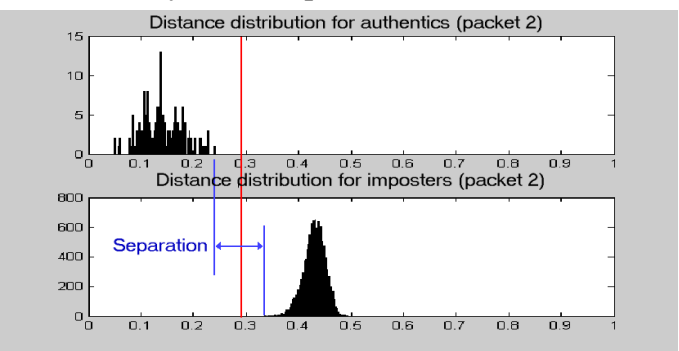

Figure 4. Distance distributions obtained for packet 2

\subsection{Packets combination}

Packets 2, 8, and 10 have independently proved to achieve positive separation. Since they capture details at different scales, they are assumed to be independent of each other. Therefore, the combination of two or three packets is likely to hold more discriminating information than what each of them does separately. We use the following combination for packets 2 and 10 :

$$
H D_{C}=\frac{1}{N} \sum_{j, k}\left|s_{2}{ }^{(1)}(j, k)-s_{2}{ }^{(2)}(j, k)\right|\left|s_{10}{ }^{(1)}(j, k)-s_{10}{ }^{(2)}(j, k)\right|(5)
$$

We multiply element-wise the coefficients of the two "difference" sub-images and normalize the result by a factor of two. As we assume that packets 2 and 10 are independent, the combined distance is the product of the distances obtained independently for packet 2 and 10, multiplied by two. For example $0.45 * 0.46 * 2=0.41$ for imposters, and $0.18 * 0.20 * 2=0.07$ for authentics. Therefore the separation is ideally increased from 0.45 $0.20=0.25$ to $0.41-0.07=0.34$. Fig. 5 confirms that the separation is effectively increased, (by a factor of 2, from 0.082 to 0.16 ). Introducing packet 8 did not yield any substantial improvements.

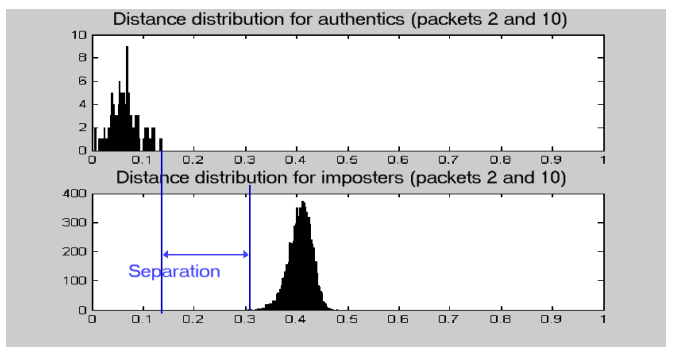

Figure 5. Distance distributions obtained for combined packets 2 and 10

\section{ROBUSTNESS OF THE ALGORITHM}

We now focus on the robustness of the proposed algorithm in relation to illumination, blurring, optical axis deviation and local defects (Fig. 6). All the possible modifications were carried out after the segmentation process. The separation for combined packets is certain to be positive if each one is positive. Therefore, we study independently the separation for wavelet packets 2,8 and 10 using the distance measure (4). (a)

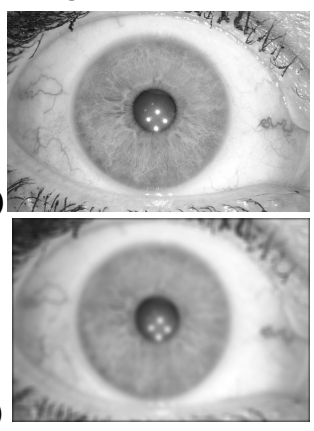

(b)

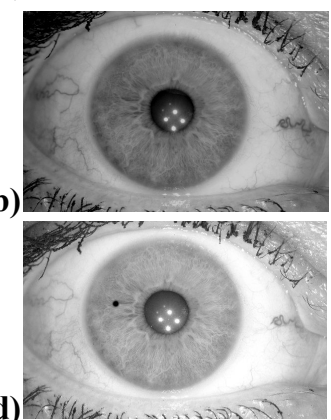

Figure 6. a) Original photo, b) increase of $20 \%$ in the dark-field illumination, c) averaging filter size of 11, d) black spot of diameter 15 pixels

\subsection{Illumination}

The illumination of images was modified in a linear manner.

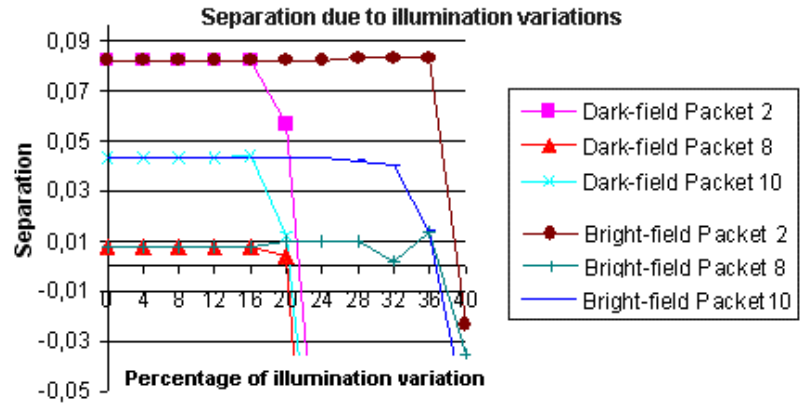

Figure 7. Separation as a function of the illumination

Fig. 7 shows that the algorithm is robust in the dark-field illumination up to an increase of $20 \%$, and in the brightfield illumination up to an increase of $36 \%$. These results are not surprising since we use an encoding based on the sign of the wavelet coefficients. The performance lost is due to a saturation effect that cancels some contrasted details.

\subsection{Optical axis deviation}

When a photo is taken, it is possible that the optical axis is not strictly orthogonal to the eye. Table 1 presents some results concerning the possible deviation of the optical axis. The tests show that the algorithm is robust for images with an optical axis deviation up to $10^{\circ}$ in both axes. 


\begin{tabular}{|c|c|c|c|c|}
\hline \multicolumn{2}{|c|}{$\begin{array}{c}\text { Optical axis } \\
\text { deviation (degrees) }\end{array}$} & \multicolumn{3}{|c|}{ Separation } \\
\hline Axis X & Axis Y & Packet 2 & Packet 8 & Packet 10 \\
\hline 0 & 0 & 0,08 & 0,01 & 0,04 \\
\hline 2,5 & 10 & 0,11 & 0,01 & 0,01 \\
\hline 5 & 5 & 0,07 & 0,01 & 0,08 \\
\hline 7,5 & 7,5 & 0,09 & 0,01 & 0,05 \\
\hline 7,5 & 10 & 0,11 & 0,00 & 0,04 \\
\hline 10 & 7,5 & 0,09 & 0,00 & 0,04 \\
\hline 10 & 10 & 0,08 & 0,01 & 0,03 \\
\hline 12,5 & 12,5 & 0,06 & $-0,02$ & 0,01 \\
\hline 15 & 15 & 0,04 & 0,00 & $-0,04$ \\
\hline 17,5 & 17,5 & 0,00 & $-0,05$ & $-0,07$ \\
\hline
\end{tabular}

Table 1. Separation as a function of variation of the optical axis

\subsection{Blurring}

An average filter was applied to the images. From Fig. 8, it is noticeable that the algorithm is very robust, up to an average filter size of 17 .

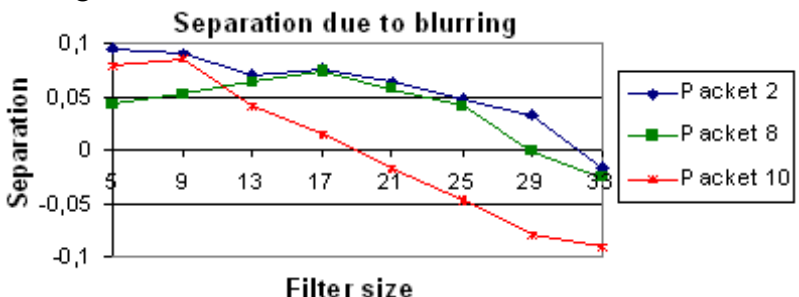

Figure 8. Separation as a function of the blurring filter size

\subsection{Local defects}

A database of 18 images was used in this case. We put a black or white spot anywhere in the iris to simulate a local defect, such as a light reflect. The results in Fig. 9 show that the algorithm can support a black spot diameter up to 15 pixels.

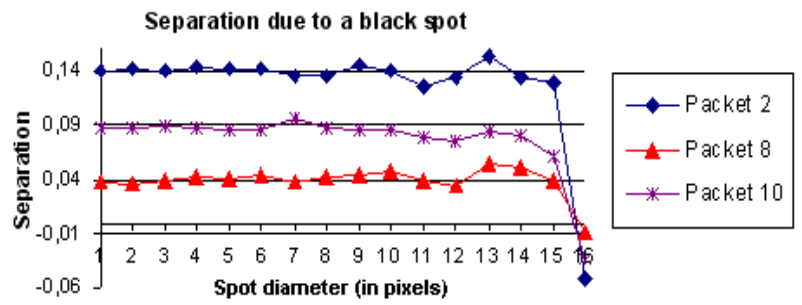

Figure 9. Separation as a function of the spot size

The algorithm seems to be more sensitive to a white spot since it can only support a white spot diameter up to 9 pixels. Combining local distance measures rather than computing a global distance measure could allow to detect the location of a possible defect and to discard the corresponding signature part in the decision algorithm. Thus, better robustness could be achieved.

\section{CONCLUSION AND FUTURE WORK}

A wavelet packet approach to iris recognition has been proposed and evaluated. We have shown that three packets could be used to generate signatures that allow to correctly identify in our database all authentic irises and dismiss all imposters. This is verified by computing the separation between the largest authentic distance and the smallest imposter distance. The packets, that carry most texture information, have been identified through an energy measure. We have also shown that the discrimination can be increased by a factor of two by combining two packets. Another contribution of this paper resides in the evaluation of the robustness of the proposed algorithm. The tests show that it is robust in relation to illumination, blurring, optical axis deviation, and, to a lesser extent, to local defects. Future work will include the extension of our database in order to conduct further research about packet combination and distance measure and ultimately improve our current results.

\section{REFERENCES}

[1] "Biometric Personal Identification System Based on Iris Analysis.", U.S. Patent No. 5,291,560 issued March 1, 1994 (J. Daugman)

[2] J. Daugman, "High confidence visual recognition of persons by a test of statistical independence.", IEEE Trans. PAMI, vol. PAMI-15, pp. 1148-1161, 1993

[3] Y. Zhu, T. Tan and Y. Wang, "Biometric personal identification based on iris patterns", Proc. 15th Int. Conf. on Pattern Recognition, Barcelona, Spain, 2000

[4] Z. Sun, Y. Wang, T. Tan, J. Cui, "Cascading Statistical and Structural Classifiers for Iris Recognition", ICIP Conf., Singapore, 2004.

[5] Tisse et al., "Person Identification technique using human iris recognition", Proc.15th Int. Conf. on Vision Interface, Calgary, Canada, 2002

[6] R. P. Wildes, "Iris recognition: an emerging biometric technology", Proc. IEEE 85, Vol 9, pp. 1348-1363, 1997

[7] C. Sanchez-Avila, R. Sanchez-Reillo, "Two different approaches for iris recognition using Gabor filters and multiscale zero-crossing representation", Pattern Recognition Vol 38, pp. 231-241, 2005

[8] S. Lim et al., "Efficient iris recognition through improvement of feature vector and classifier", ETRI J., Vol 23, pp. 61-70, 2001

[9] J. Bergh, F. Ekstedt and M. Lindberg, Wavelets, Studentlitteratur, Lund, 1999

[10] E. Rydgren, T. Ea, F. Amiel, F. Rossant and A. Amara, "Iris features extraction using wavelet packets", ICIP Conf., Singapore, 2004

[11] E. Krichen et al., "Iris Identification Using Packet for Images in Visible Light Illumination", Conference, ICBA, Hong Kong, China, July 15-17, 2004

[12] Miles Research, www.milesresearch.com

[13] M. Misiti et al. , "Les ondelettes et leurs applications" Ed. Hermès, 2003. 